\section{Case Reports in Ophthalmology}

\title{
Recurrent Vitreous Hemorrhage from an Optic Nerve Retinal Arterial Macroaneurysm
}

\author{
Jessica F. Yang ${ }^{a} \quad$ Kamal Kishore ${ }^{a, b}$ \\ ${ }^{a}$ University of Illinois College of Medicine, Peoria Campus, Peoria, IL, USA; ${ }^{\mathrm{b}}$ Illinois Retina \\ and Eye Associates, Peoria, IL, USA
}

\section{Keywords}

Endolaser · Optic nerve retinal arterial macroaneurysm - Vitreous hemorrhage $\cdot$ Bevacizumab

\begin{abstract}
Objective: To report a case of recurrent vitreous hemorrhage from an optic nerve retinal arterial macroaneurysm (ONRAM) successfully treated with intraoperative endolaser. Patient and Methods: A 92-year-old woman on oral aspirin and warfarin anticoagulation for atrial fibrillation developed three episodes of dense vitreous hemorrhage from an ONRAM. Due to failure of the vitreous hemorrhage to clear spontaneously, a total of three pars plana vitrectomy (PPV) procedures were performed along with a $1.25-\mathrm{mg}$ intravitreal bevacizumab injection after the third episode of hemorrhage. During the third PPV procedure, a 25-gauge 532-nm green diode laser endoprobe was used to deliver low-power $(100 \mathrm{~mW})$ and longduration (500 ms) laser spots directly on the ONRAM to induce intraoperative shrinkage of the ONRAM. Results: After the endolaser treatment, the macroaneurysm showed involution due to fibrosis without any adverse effects on retinal circulation or visual field defect. No recurrence of vitreous hemorrhage was noted after 2 years of follow-up. Conclusion: Oral anticoagulant use may have been responsible for the atypical clinical course in our patient. Laser photocoagulation, including intraoperative endolaser photocoagulation, may be considered in selected cases of symptomatic ONRAMs.




\section{Case Reports in Ophthalmology}

\section{Introduction}

A retinal arterial macroaneurysm (RAM) is an outpouching of the retinal arteriolar wall within the first three bifurcations of the central retinal artery [1]. RAMs are most commonly seen in the sixth decade, have a female preponderance, and are commonly associated with hypertension and hyperlipidemia [2,3]. The overall incidence of RAM is 1 in 4,500 individuals over the age of 40 [4], with only $3-8 \%$ of these cases occurring over the optic nerve (ONRAM) [2, 3]. The natural history of ONRAMs is not well understood due to their rarity. ONRAMs can be an asymptomatic incidental finding [5] or can result in vitreous hemorrhage [6-9], branch retinal artery occlusion [8, 10-12], or macular edema [13-16]. To our knowledge, only five eyes in three publications required pars plana vitrectomy (PPV) for vitreous hemorrhage from a ruptured ONRAM [7-9]. In each case, vitreous hemorrhage was a one-time event and was followed by spontaneous involution of the ONRAM without any treatment. Herein, we report three episodes of dense vitreous hemorrhages from an ONRAM in a patient on oral anticoagulants.

\section{Case Report}

A 92-year-old pseudophakic female with intermediate age-related macular degeneration presented with a 1-day history of sudden loss of vision in her left eye (OS). Her past medical history included chronic atrial fibrillation, hypertension, and hypercholesterolemia. Her medications included aspirin $81 \mathrm{mg} /$ day and warfarin (Coumadin ${ }^{\circledR}$ ) with a therapeutic international normalized ratio maintained between 2 and 3. On examination, the visual acuity in the right eye (OD) was 20/30 and in OS it was light perception. Intraocular pressure (IOP) was $16 \mathrm{~mm} \mathrm{Hg} \mathrm{OD} \mathrm{and} 45 \mathrm{~mm} \mathrm{Hg}$ OS by Goldmann applanation tonometry. Slit-lamp examination in OD showed a well-centered posterior chamber intraocular lens, and in OS showed red blood cells in the anterior chamber and a well-centered posterior chamber intraocular lens in the capsular bag with an intact posterior capsule. Dilated fundus examination in OD showed changes of intermediate dry age-related macular degeneration, and in OS it showed a dense vitreous hemorrhage blocking the red fundus reflex. B-scan ultrasonography OS showed dense vitreous hemorrhage without retinal detachment or suprachoroidal hemorrhage. The patient was started on timolol-dorzolamide (Cosopt ${ }^{\circledR}$ ) and brimonidine drops. Six days after initial presentation, a transconjunctival 23-gauge PPV was performed due to failure of hemorrhage to clear and persistently elevated IOP. Intraoperatively, we discovered a large pulsatile macroaneurysm over the optic nerve without any associated vitreous attachment or traction to the RAM. The patient had complete posterior vitreous detachment. The ONRAM was not treated at this time. Her postoperative course was uneventful, with improvement of visual acuity OS 2 weeks after the procedure to 20/40 and return of IOP to normal.

One month after her initial vitrectomy, the patient presented with sudden loss of vision OS due to recurrent dense vitreous hemorrhage that reduced her visual acuity to hand motions and completely obscured the view of the retina. IOP was normal. A repeat B-scan ultrasonography confirmed dense vitreous hemorrhage and showed an attached retina. A transconjunctival 25-gauge PPV was performed 5 weeks later due to failure of hemorrhage to clear spontaneously. During this vitrectomy, the pulsatile macroaneurysm over the optic nerve was unchanged and was not treated due to the well-known natural history of RAMs to involute spontaneously following their rupture [3]. Two weeks after the second PPV, the 


\section{Case Reports in Ophthalmology}

Case Rep Ophthalmol 2017;8:503-509

DOI: $10.1159 / 000481704$

C 2017 The Author(s). Published by S. Karger AG, Basel www.karger.com/cop

Yang and Kishore: Recurrent Vitreous Hemorrhage from an Optic Nerve Retinal Arterial Macroaneurysm

patient's OS vision improved to her baseline of 20/30, and dilated fundus examination showed clear ocular media and a large ONRAM (Fig. 1).

Three weeks after her second PPV, the patient presented with a third episode of sudden loss of vision OS from a dense vitreous hemorrhage that reduced her vision to hand motions and once again completely obscured the view of the retina. IOP was normal. A repeat B-scan ultrasonography was performed and confirmed an attached retina. After obtaining informed consent, $1.25 \mathrm{mg} / 0.05 \mathrm{~mL}$ intravitreal bevacizumab (Avastin ${ }^{\circledR}$ ) was administered off-label under topical anesthesia. The vitreous hemorrhage failed to clear after 6 weeks of observation and her visual acuity remained at hand motions OS. IOP remained normal, and there was no view of retinal structures during 6 weeks of observation following intravitreal Avastin injection. Discontinuation of anticoagulation was discussed, but her medical doctor advised against it. A 25-gauge PPV was performed to clear the hemorrhage. Intraoperatively, the same pulsatile ONRAM was observed and was unchanged from its initial appearance during the first PPV performed 18 weeks earlier. A 25-gauge straight endolaser probe was brought close to the ONRAM, almost touching it, and 71 laser spots from a 532-nm wavelength green diode laser (Iridex Corp., Mountain View, CA, USA) with a power intensity of $100 \mathrm{~mW}$ and a duration of $500 \mathrm{~ms}$ were applied directly to the ONRAM in a "painting" manner to gently cover the aneurysm and cause mild shrinkage.

Postoperatively, fibrosis and involution of the ONRAM were noted, with no evidence of retinal vascular occlusion (Fig. 2a). An intravenous fluorescein angiogram 10 weeks after the third vitrectomy showed a hypofluorescent mass over the nerve consistent with lack of blood flow within the ONRAM (Fig. 2b). The patient maintained 20/30 vision OS without any visual field defect or optic atrophy over 2 years of follow-up, with complete fibrosis of the ONRAM despite continued use of oral anticoagulants (Fig. 3).

\section{Discussion}

RAMs are typically found on the first three orders of the arteriolar tree and are rarely located on the optic disc [1-3]. The differential diagnosis of a mass lesion on the disc includes granulomatous inflammatory lesions such as sarcoidosis and toxoplasmosis as well as tumors such as capillary hemangioma, astrocytoma, melanocytoma, melanoma, and metastatic tumor [10]. The diagnosis of ONRAM was established in our case by its typical orange red appearance and pulsatile nature [9]. ONRAM can be symptomatic from exudation or rupture. Compared to RAMs located outside of optic nerve, branch retinal arteriolar occlusion following rupture of an ONRAM resulting in secondary closure of distal vasculature seems to be a disproportionately common event, having been reported in 5 of 18 reported cases of ONRAMs $[8,10-12,17]$. Subhyaloid or vitreous, but not submacular, hemorrhage following rupture of ONRAMs has been reported in 8 eyes in five publications [6-9, 18]. It cleared spontaneously in 3 eyes $[6,7,18]$, while 5 eyes in three publications required vitrectomy [7-9]. Spontaneous involution of ONRAM occurred in each of 8 eyes with vitreous or subhyaloid hemorrhage. One patient was treated with discontinuation of aspirin [6]. Anticoagulation status in another 7 eyes is not known. Notably, recurrence of vitreous hemorrhage was not observed in any eye. Our patient was taking both aspirin and warfarin, with a therapeutic international normalized ratio maintained between 2 and 3. Since involution of RAM is due to thrombosis within the aneurysm followed by fibrosis [3], it is reasonable to speculate that failure of spontaneous involution of the ONRAM in our patient was most likely due to anticoagulant use. 
Macular edema secondary to exudation from an ONRAM has been reported in 6 eyes of five publications. One eye was treated with focal laser of the ONRAM [14], one with posterior subtenon steroid injection [16], and the remaining 4 eyes resolved spontaneously [13$15,19]$.

Intravitreal anti-VEGF injections have been shown to be effective in the management of complications of RAMs [20]. Our patient was given an intravitreal bevacizumab injection after the third episode of vitreous hemorrhage, but did not show improvement after 6 weeks of observation. Whether enhanced drug clearance in a vitrectomized eye contributed to the lack of efficacy of intravitreal bevacizumab in our patient cannot be determined at this time. To our knowledge, there is no published report regarding the efficacy of intravitreal antiVEGF injections for ONRAMs.

Laser photocoagulation of RAM, first described by Lewis et al. [21], carries the risk of several complications, such as vascular occlusion and rupture of RAM. Laser treatment on or near the optic nerve carries an additional risk of visual field defect due to damage to the optic nerve [22]. Khan et al. [14] employed an argon green laser (100-200 $\mu$ m diameter, $200 \mathrm{~mW}$ power, $0.1 \mathrm{~s}$ duration, 162 spots) delivered at the slit-lamp to successfully treat an ONRAM in a patient with worsening visual acuity due to macular edema. In our case, we utilized a 25-gauge endolaser probe under direct visualization of an operating microscope to induce shrinkage of the ONRAM, which ultimately led to its fibrosis and involution. A painting technique was used to gently cover the aneurysm using a low power of $100 \mathrm{~mW}$ and long duration $(500 \mathrm{~ms}$ ) setting, avoiding intense application of laser energy at any one spot, which can cause rupture of the aneurysm. Unlike universal occurrence of central or arcuate scotoma observed by Garcia-Arumi et al. [22] following laser treatment of papillary capillary hemangiomas, no field defect or optic atrophy was noted over 2 years of follow-up. The laser energy used by us and by Khan et al. [14] was considerably less compared to that employed by Garcia-Arumi et al. [22]. Experimental studies have shown that power intensity of $<400 \mathrm{~mW}, 0.2 \mathrm{~s}$ duration, and $100 \mu \mathrm{m}$ spot size is unlikely to damage the optic nerve even when the laser is directly applied to the nerve itself [23]. Although no intraoperative complications were noted, bleeding from rupture of RAM during endolaser treatment could have been easily managed with a temporary elevation of IOP. Thus, intraoperative endolaser treatment of ONRAMs during PPV may be a safer approach than slit-lamp-based laser delivery in the postoperative period.

To conclude, we report an unusual case of ONRAM that led to three episodes of dense vitreous hemorrhage in a 92-year-old female on oral anticoagulants. The patient failed to respond to intravitreal bevacizumab injection administered 1 day after her third episode of vitreous hemorrhage. Endolaser treatment during her third PPV led to successful fibrosis of the ONRAM with no side effects. To our knowledge, this is the only reported case of ONRAM treated with an intraoperative endolaser. Our report suggests that intraoperative endolaser photocoagulation utilizing low-power and long-duration laser applications in a painting manner may be an effective treatment strategy for selected cases of ONRAM.

\section{Statement of Ethics}

All procedures were done in accordance with the tenets of the Helsinki Declaration of 1964, as revised in 2013. Institutional review board approval was not required for this retrospective study involving a single case. 


\section{Disclosure Statement}

The authors have no financial disclosures to make and no proprietary interests to disclose. There are no conflicts of interest for any of the authors.

\section{References}

1 Robertson DM: Macroaneurysms of the retinal arteries. Trans Am Acad Ophthalmol Otolaryngol 1973;77:0P55-0P67.

2 Palestine AG, Robertson DM, Goldstein BG: Macroaneurysms of the retinal arteries. Am J Ophthalmol 1982;93:164-171.

-3 Rabb MF, Gagliano DA, Teske MP: Retinal arterial macroaneurysms. Surv Ophthalmol 1988;33:73-96.

4 Xu L, Wang Y, Jonas JB: Frequency of retinal macroaneurysms in adult Chinese: the Beijing Eye Study. Br J Ophthalmol 2007;91:840-841.

5 Kowal L, Steiner H: Arterial macroaneurysm of the optic disc. Aust NZ J Ophthalmol 1991;19:75-77.

-6 Quhill F, Smith JM, Scotcher SM: Arterial macroaneurysm on the optic disc. Eye (Lond) 2004;18:321322.

7 Hirano K, Mitamura Y, Ogata K, et al: Three cases of retinal arterial macroaneurysm on the optic disc. Nippon Ganka Gakkai Zasshi 2010;114:801-804.

8 Kubo E, Kimura K, Sugimoto Y, et al: Bilateral optic disc macroaneurysm associated with acquired vascular loop. Jpn J Ophthalmol 2009;53:561-563.

-9 Takeyama M, Iwaki M, Zako M: Macroaneurysm on the optic disc in a patient with aortic dissection. Case Rep Ophthalmol 2013;4:234-237.

$>10$ Brown GC, Weinstock F: Arterial macroaneurysm on the optic disk presenting as a mass lesion. Ann Ophthalmol 1985;17:519-520.

-11 Ben Yahia S, Jenzeri S, Jelliti B, et al: An unusual complicated cilioretinal artery macroaneurysm on the optic disc. Int Ophthalmol 2009;29:507-509.

12 Das-Bhaumik RG, Lindfield D, Quinn SM, et al: Optic disc macroaneurysm in evolution: from incidental finding to branch retinal artery occlusion and spontaneous resolution. Br J Ophthalmol 2011;95:145146, 155.

13 Ichibe M, Oya Y, Yoshizawa T, et al: Macroaneurysm on the optic disk associated with congenital retinal arterial malformation. Retina 2004;24:985-986.

14 Khan BA, Desai UR, Khan KA, et al: Retinal arteriolar macroaneurysms overlying the optic nerve. Retin Cases Brief Rep 2009;3:253-258.

-15 Rey A, Alforja S, Sabater N, et al: Natural history of an optic disc macroaneurysm. Arch Soc Esp Oftalmol 2014;89:113-116.

-16 Nagai N, Ohta Y, Izumi-Nagai K, et al: Sneddon's syndrome with optic disc macroaneurysm and macular edema successfully treated with subtenon steroid injection. Acta Ophthalmol 2016;94:e517-e519.

-17 Mitamura Y, Miyano N, Suzuki Y, et al: Branch retinal artery occlusion associated with rupture of retinal arteriolar macroaneurysm on the optic disc. Jpn J Ophthalmol 2005;49:428-429.

18 Rahimy E, Doyle BC, Brown GC: Ruptured retinal arterial macroaneurysm on the optic disk. Retin Cases Brief Rep 2017;11:12-14.

19 Toyama T, Kato S, Noda Y, et al: Spontaneous resolution of a macroaneurysm on the optic disc. Int J Ophthalmol 2014;7:179-180.

20 Kishore K: Long-term management of complications of retinal artery macroaneurysms with intravitreal aflibercept injection. Case Rep Ophthalmol 2016;7:162-171.

-21 Lewis RA, Norton EW, Gass JD: Acquired arterial macroaneurysms of the retina. Br J Ophthalmol 1976;60:21-30.

22 Garcia-Arumi J, Sararols LH, Cavero L, et al: Therapeutic options for capillary papillary hemangiomas. Ophthalmology 2000;107:48-54.

-23 Apple DJ, Wyhinny GJ, Goldberg MF, et al: Experimental argon laser photocoagulation. II. Effects on the optic disc. Arch Ophthalmol 1976;94:296-304. 


\section{Case Reports in Ophthalmology}

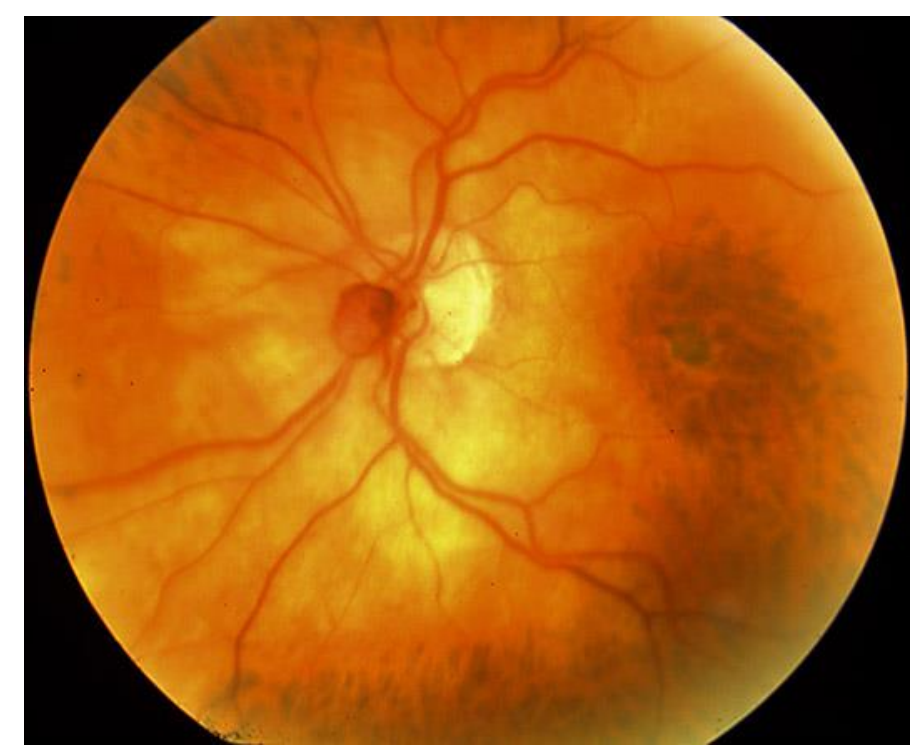

Fig. 1. Fundus photograph of the left eye 2 weeks after the second vitrectomy showing a large retinal arterial macroaneurysm over the nerve.
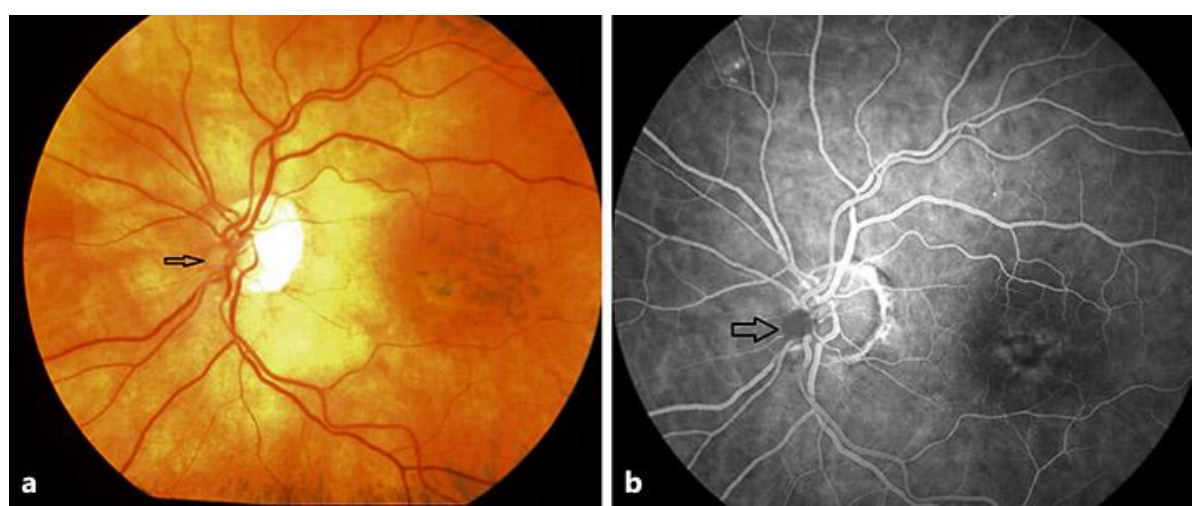

Fig. 2. a Fundus photograph of the left eye 10 weeks after the third vitrectomy showing a partially fibrosed retinal arterial macroaneurysm over the optic nerve (arrow). b Intravenous fluorescein angiogram 10 weeks after the third vitrectomy at $35 \mathrm{~s}$ showing normal retinal circulation. A hypofluorescent mass representing thrombosed optic nerve macroaneurysm is visible on the optic nerve (arrow). 


\section{Case Reports in Ophthalmology} Case Rep Ophthalmol 2017;8:503-509 DOI: $10.1159 / 000481704$

C 2017 The Author(s). Published by S. Karger AG, Basel www.karger.com/cop

Yang and Kishore: Recurrent Vitreous Hemorrhage from an Optic Nerve Retinal Arterial Macroaneurysm

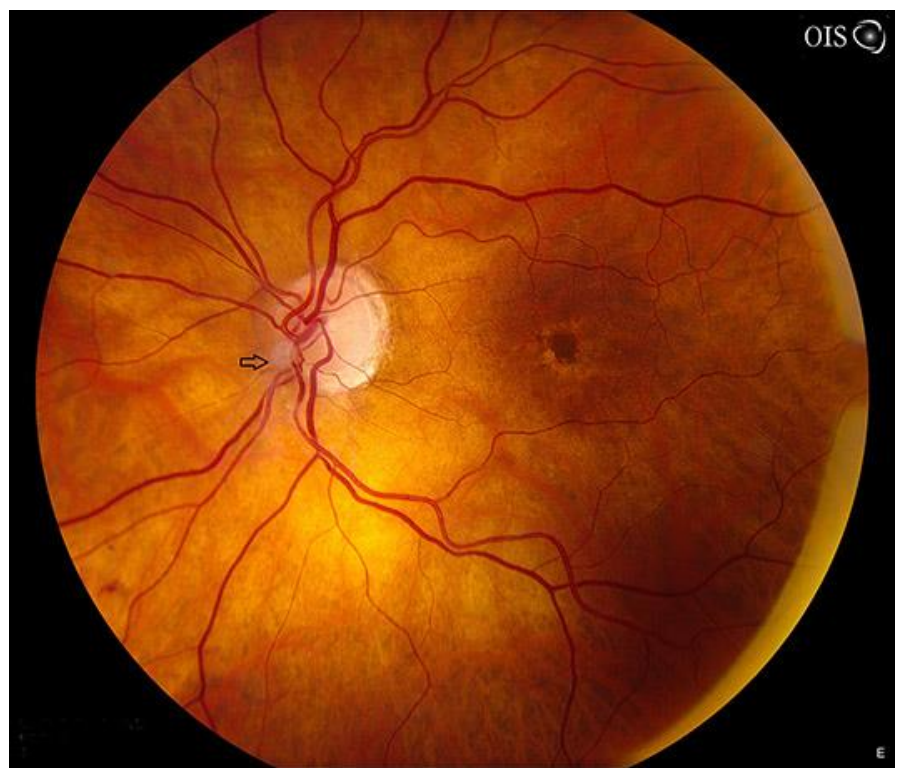

Fig. 3. Fundus photograph of the left eye 17 months after the third vitrectomy showing a completely fibrosed retinal arterial macroaneurysm over the nerve (arrow). The macula shows changes of atrophic agerelated macular degeneration. 